\title{
A Simple Model for Induction Core Voltage Distributions
}

\author{
Richard J. Briggs (SAIC) and William M. Fawley (LBNL)
}

\section{Introduction}

In fall 2003 T. Hughes of MRC used a full EM simulation code (LSP) to show that the electric field stress distribution near the outer radius of the longitudinal gaps between the four Metglas induction cores is very nonuniform in the original design of the DARHT-2 accelerator cells. In this note we derive a simple model of the electric field distribution in the induction core region to provide physical insights into this result. The starting point in formulating our model is to recognize that the electromagnetic fields in the induction core region of the DARHT-2 accelerator cells should be accurately represented within a quasi-static approximation because the timescale for the fields to change is much longer than the EM wave propagation time. The difficulty one faces is the fact that the electric field is a mixture of both a "quasi-magnetostatic field" (having a nonzero curl, with Bdot the source) and a "quasi-electrostatic field" (the source being electric charges on the various metal surfaces).

We first discuss the EM field structure on the "micro-scale" of individual tape windings in Section 2. The insights from that discussion are then used to formulate a "macroscopic" description of the fields inside an "equivalent homogeneous tape wound core region" in Section 3. This formulation explicitly separates the nonlinear core magnetics from the quasi-electrostatic components of the electric field. In Section 4 a physical interpretation of the radial dependence of the electrostatic component of the electric field derived from this model is presented in terms of distributed capacitances, and the voltage distribution from gap to gap is related to various "equivalent" lumped capacitances.

Analytic solutions of several simple multi-core cases are presented in Sections 5 and 6 to help provide physical insight into the effect of various proposed changes in the geometrical parameters of the DARHT-2 accelerator cell. Our results show that over most of the gap between adjacent cores there will be near equipartition of the voltages $B U T$ there will be a region near the outer radius of each core where the voltages (and more importantly, the electric field stress) can deviate significantly from equipartition. In Section 7 we apply our results to some multicore measurements form the LBNL test stand and make some predictions for the general DARHT-2 accelerator cell configuration.

\section{A "micro-picture" of the core fields}

The DARHT-2 cores are composed of 4"-wide Metglas tape with a nominal thickness of 0.8 mils. The tape is wound with mylar insulation of nominal thickness 0.2 mils separating adjacent windings. Inside the Metglas, the electric field normal to the interface 
( $x$ direction in a planar approximation $-x$ becomes $r$ later) is negligible. This field is shorted out by the Metglas conductivity ( $\sigma^{-1} \approx 1.25 \times 10^{-6}$ ohm-meters) on the timescale $\epsilon / \sigma$ for surface charges to build up on the interface.

The magnetizing current flowing around the entire core creates an $H_{\theta}$ field $\left(H_{y}\right.$ in a planar approximation) that is constant in both $z$ and $x$ throughout each mylar sheet (see Fig. 1). We first assume the magnetic skin depth is much larger than the Metglas thickness $d_{M}$, and take both $H_{y}$ and $B_{y}=B_{M}$ as constant throughout each Metglas tape winding as well. Inside the each Metglas winding, Maxwell's equations require

$$
\frac{\partial E_{z, M}}{\partial x}=\frac{\partial B_{M}}{\partial t} \equiv \dot{B}_{M}
$$

The net current in the $z$ direction (across the tape) in any Metglas tape section must be very small --- otherwise the free charge at the ends of the tape would rapidly build up.

Taking $x=0$ at the center of a selected piece of tape, we then have

$$
E_{z}=\dot{B}_{M} x
$$

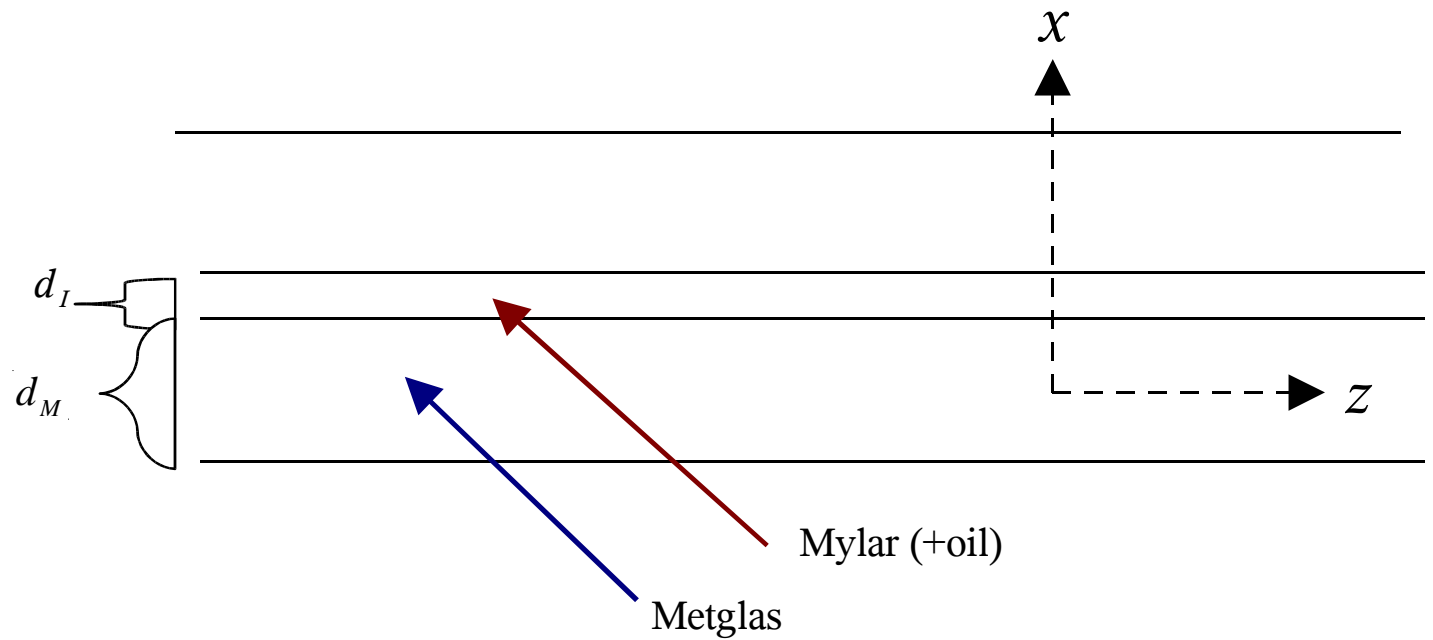

Fig. 1 - Adopted planar geometry

Since this solution to very high order is exactly the same as in adjacent Metglas layers, $E_{z}$ must vary with $x$ in the periodic fashion shown in Fig. 2 from layer to layer. Here a linear variation in $x$ through the mylar is presumed. In the mylar (subscript $I$ ), where the magnitude of $B$ is very small in comparison with its value in the Metglas, curl $E$ is approximately zero. Consequently,

$$
\frac{\partial E_{x, I}}{\partial z}=\frac{\partial E_{z, I}}{\partial x}=-\frac{d_{M}}{d_{I}} \dot{B}_{M}
$$


where $E_{x, I}$ is the electric field in the mylar normal to the interface.

At the axial ends of each inductive core, the "smooth" radial electric field in the gap region between adjacent cores, or between a core and an end plate, should be set equal to the mean value of the $x$-directed field through the layers of Metglas and mylar tape,

$$
\left\langle E_{x}\right\rangle=E_{x, I} \times \frac{d_{I}}{d_{M}+d_{I}}
$$

Using Eq. (2.3), we have

$$
\frac{\partial\left\langle E_{x}\right\rangle}{\partial z}=-\frac{d_{M}}{d_{M}+d_{I}} \dot{B}_{M}=-p_{f} \dot{B}_{M}
$$

with the last equality defining the "packing factor" $p_{f}$. Since the magnetic field in any individual Metglas winding layer is constant in $z,\left\langle E_{x}\right\rangle$ must vary linearly in $z$ across the layer width $w$.

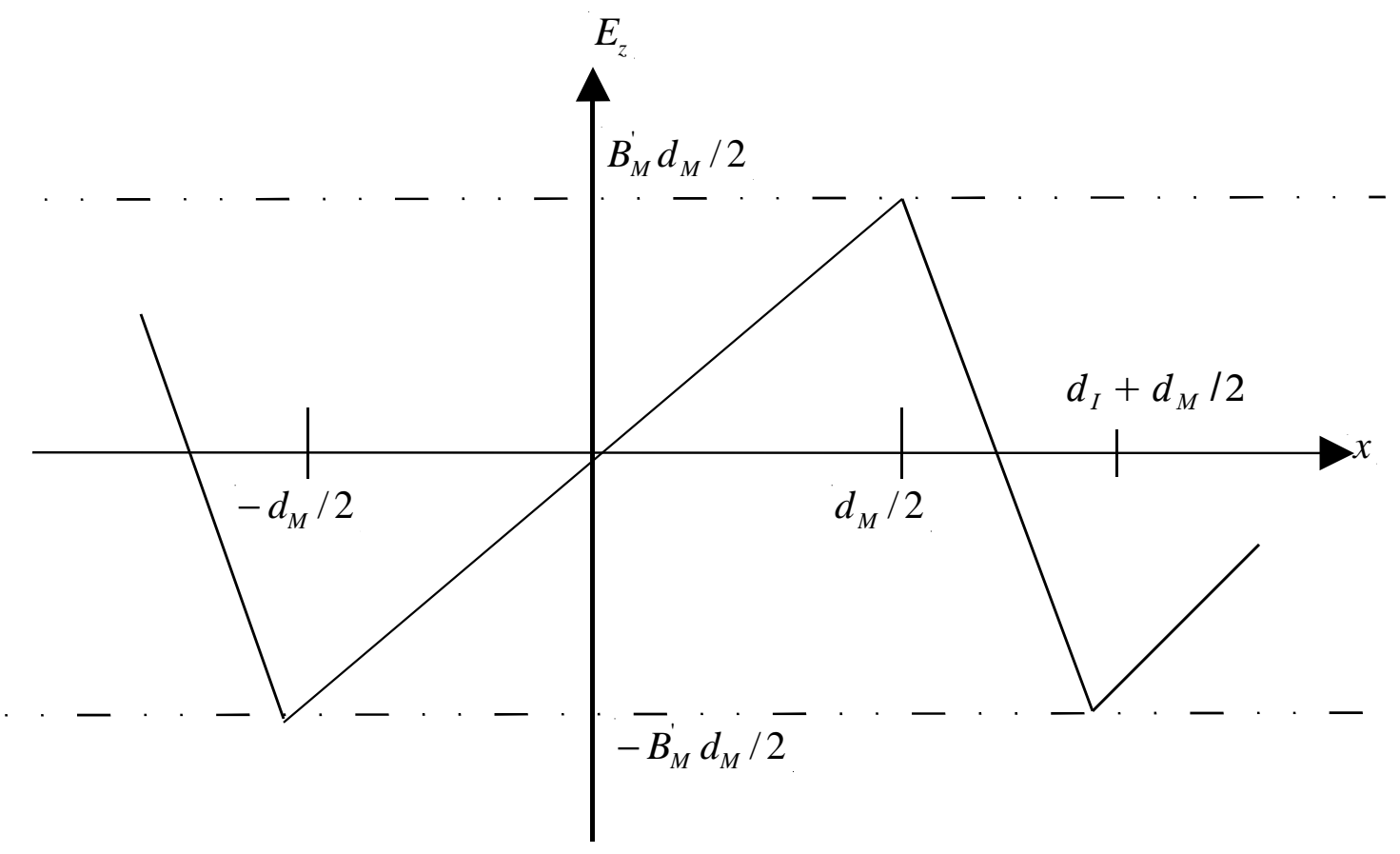

Fig. 2 Axial electric field vs. $\mathrm{x}$

In moving over to a "macro" description of the EM fields, note that the maximum value of $E_{z}$ (see Fig. 2) is of order $d_{M} / w \sim 10^{-4}$ compared to $\left\langle E_{x}\right\rangle$. We can therefore safely ignore the z-directed electric field in a "macro-picture", even though it is a critical factor in the "micro-picture" of the rapidly alternating fields across the layers. 
Also note that in a macroscopic description, the equivalent radial dielectric constant for calculating the radial displacement current using the mean radial electric field (Eq. 2.4) is

$$
\epsilon_{R}=\frac{\epsilon_{I}}{1-p_{f}}
$$

We have implicitly modeled the tape sections as individually-nested cylinders instead of the actual continuous helical winding of $\sim 20,000$ layers. This approximation is valid as long as the total radial displacement current is much larger than the real current in the helical windings. Near the outer radius of the DARHT-2 cores, the layer-to-layer capacitance across the 0.2 mil mylar sheet is $\sim 2.5$ microfarads. The resistance of one turn of the Metglas tape at the outer radius is about $3 \mathrm{ohms}$, and this impedance alone is sufficient to make the real current much less than the "capacitive" current on the 1-2 microsecond timescale. Estimating an inductive component of the layer to layer impedance is more difficult, since the axial flux between each layer is created by the sum of all the layers azimuthal current (i.e., the mutual inductance must be included). A crude estimate of the "equivalent" inductive impedance retarding the real current flow shows that it is 100-1,000 times larger than the resistive impedance when $\mu$ is large, so the real helical current is insignificant in DARHT-2. We note that in the small 7" radius core experiments at LBNL, a similar estimate of real current indicates that it may not be negligible over 2-3 microseconds when $\mu$ is small.

We now consider, as a perturbation, the variations in magnetic field across the Metglas from the "eddy currents". Adopting Eq. 2.2 as a first approximation to the axial electric field, the magnetic field variation follows from Maxwell's equations as

$$
\frac{\partial H_{y}}{\partial x}=\sigma E_{z} \approx \sigma \dot{B}_{M} x
$$

The solution is

$$
H_{y}=H_{y 0}\left\{1+\frac{\dot{B}_{M} \sigma}{2 H_{y 0}}\left(x^{2}-\frac{d_{M}^{2}}{4}\right)\right\}
$$

We define a nonlinear, time dependent permeability as

$$
\mu \equiv \frac{\dot{B}_{M}}{\frac{\partial H_{y}}{\partial t}}
$$

and a magnetizing current rate-of-rise timescale

$$
\tau \equiv \frac{H_{y}}{\frac{\partial H_{y}}{\partial t}}
$$


The variation in magnetic field across the Metglas layer is then

$$
\frac{\Delta H_{y}}{H_{y 0}} \approx \frac{\mu \sigma d_{M}^{2}}{8 \tau}
$$

Using a risetime of 2 microseconds, and a relatively high value of normalized permeability $\left(\mu / \mu_{0} \sim 10^{3}\right)$, the variation is about $2.5 \%$. These eddy-current effects can of course be incorporated into the model of the nonlinear magnetic response of the Metglas tape.

\section{An Analytic Core Model}

In this and the next few sections we present a simple model describing how the core voltages are distributed both radially within a given core and from core to core in a multicore cell. First we will examine the simple but heuristic case of a single core cell, followed by the more complicated situations of two or more cores in the following sections.

The magnetic fields in the core region are basically magnetostatic in character because the large inductance constrains the rate of rise of the magnetizing current. In our formulation we assume that the macroscopic (mean) azimuthal magnetic field $B_{\theta}=p_{f} B_{M}$ in any one core is a function of $r$ and $t$ only. Otherwise, one is free to use arbitrary models of the nonlinear magnetization including eddy currents, etc., as inputs.

Since in an equivalent homogeneous core model $E_{z}=E_{\theta}=0$, Maxwell's equations require $\partial E_{r} / \partial z=-\dot{B}_{\theta}(r, t)$. At a given radius, $B_{\theta}$ within the core is independent of $z$. Then we may separate $E_{r}$ into a term which scales linearly with $z$ and a $z$-independent term

$$
E_{r}(r, z, t)=-z \dot{B}_{\theta}(r, t)+E_{r}^{c}(r, t)
$$

and define $z=0$ to lie at the longitudinal middle of the core.

We now introduce three separate voltage quantities: $\Psi$, the "full" voltage; $V$, the "inductive" voltage; and $\Phi$, the "electrostatic" voltage. For each core, let us then define the "left" full voltage $\psi_{L}(r)$ and "right" full voltage $\psi_{R}(r)$ as

$$
\begin{aligned}
& \psi_{L}(r) \equiv-\int_{a}^{r} d r^{\prime} E_{r}\left(r^{\prime}, z=-w / 2\right) \quad \text { and } \\
& \psi_{R}(r) \equiv-\int_{a}^{r} d r^{\prime} E_{r}\left(r^{\prime}, z=+w / 2\right)
\end{aligned}
$$


Fig. 3 Electric fields around Metglas-mylar interface

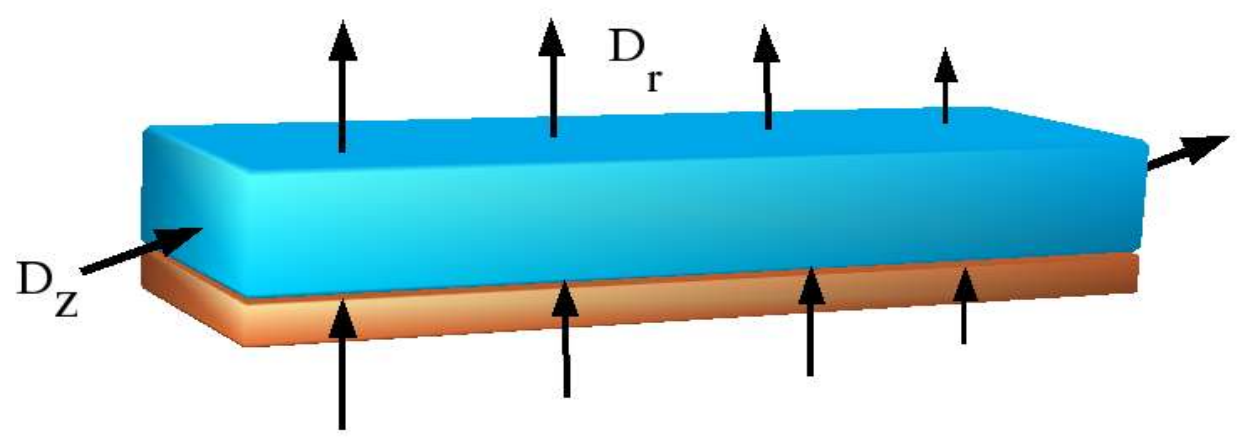

where $E_{r}$ is the "total" electric field (the sum of inductive and electrostatic components) and $a$ is the core inner radius. We then define the "inductive" voltage $V$ and the "electrostatic" voltage $\Phi$ as

$$
\begin{aligned}
& V(r)=-\int_{a}^{r} d r^{\prime} E_{R}(\text { inductive })=-w \times \int_{a}^{r} d r^{\prime} \dot{B}_{\theta} \\
& \Phi(r) \equiv-\int_{a}^{r} d r^{\prime} E_{r}^{c}\left(r^{\prime}, t\right)
\end{aligned}
$$

Therefore,

$$
\begin{aligned}
& \psi_{L}(r)=\Phi(r)+V(r) / 2 \\
& \psi_{R}(r)=\Phi(r)-V(r) / 2
\end{aligned}
$$

Note that $\psi_{L}(r)-\psi_{R}(r)=V(r)$ which is equivalent to $-\int E \cdot d l$ presuming that $E_{z}$ is identically zero along the integral path (i.e. a Metglas layer). Moreover, we have not placed any conditions upon either $V(r)$ or $\Phi(\mathrm{r})$. This permits $V(r)$ to handle a $\dot{B}_{\theta}$ which varies significantly with $r$ as would be true for a $b / a$ ratio exceeding 2 (as in DARHT-2) or due to saturation effects.

The source term for $\Phi$ will be the free charges which redistribute themselves on each Metglas layer to short out both the internal $E_{\theta}$ and $E_{z}$. Applying Gauss's law (see Fig. 3 ) to the fields surrounding one composite layer of Metglas + Mylar, we have $\oint \bar{D} \cdot d \bar{A}=Q=0$. The net charge on the left and right edge of the Metglas facing the oil-filled gap is $2 \pi r d_{T} \epsilon_{G}\left(\left\langle E_{z, R}\right\rangle-\left\langle E_{z, L}\right\rangle\right)$ where $\epsilon_{G}$ is the dielectric constant for the oil, $d_{T} \equiv d_{M}+d_{I}$ and the angle brackets mean a "coarse" averaging many $d_{T}$ thicknesses away in $\mathrm{z}$ from the edge of the core. The net charge on the upper and lower radial surfaces of a Metglas layer is

$$
2 \pi \epsilon_{I} \int_{-w / 2}^{+w / 2}\left\{r_{u} E_{r}\left(r_{u}, z\right)-r_{l} E_{r}\left(r_{l}, z\right)\right\} d z=-2 \pi w \epsilon_{I} d_{T} \frac{\partial}{\partial r}\left\{r \frac{d_{T}}{d_{I}} \frac{\partial \Phi}{\partial r}\right\}
$$

where $r_{u, l} \equiv r \pm d_{T} / 2$ and the radial derivative of $\Phi$ refers to the average gradient from the middle of one mylar layer to the next (which is smaller than the peak gradient by a factor of $\left(1-p_{f}\right)$--- see Eq. 2.4). Solving, we find the important result 


$$
\Delta E_{z}=\left(\frac{\epsilon_{R}}{\epsilon_{G}}\right) w \frac{1}{r} \frac{\partial}{\partial r} r \frac{\partial \Phi}{\partial r}
$$

where $\epsilon_{R} \equiv \epsilon_{I} /\left(1-p_{f}\right)$ and $\Delta E_{z} \equiv E_{z, R}-E_{z, L}$.

To determine a final equation for $\Phi$, we must relate it to $\Delta E_{z}$. For the single core case this is rather straight-forward. As shown in Fig. 4, there is a drive plate to the right of the inductive core with a voltage $V_{D}$. Both to the left and to the top of the core there is a "ground" plane to which we assign a zero voltage. Everywhere outside the core, $E$ is curlfree and thus we may define $E=-\nabla \Phi$. Consequently, for gap widths $\Delta_{1,2} \ll(b-a)$

$$
\begin{aligned}
& E_{z, L}(r)=-\frac{\Psi_{L}(r)}{\Delta_{1}}=-\frac{V(r)}{2 \Delta_{1}}-\frac{\Phi(r)}{\Delta_{1}} \\
& E_{z, R}(r)=+\frac{\Psi_{R}(r)}{\Delta_{2}}=-\frac{V(r)}{2 \Delta_{2}}+\frac{\Phi(r)}{\Delta_{2}}
\end{aligned}
$$

Using these results in Eq. 3.6, we find

$$
\frac{1}{r} \frac{\partial}{\partial r} r \frac{\partial \Phi}{\partial r}-2 \kappa^{2} \Phi=2 \kappa^{2}\left(\frac{\Delta_{2}-\Delta_{1}}{\Delta_{1}+\Delta_{2}}\right) \frac{V(r)}{2}
$$

where we have defined

$$
\kappa^{2} \equiv \frac{\epsilon_{G}}{\epsilon_{R}} \times \frac{\Delta_{1}+\Delta_{2}}{2 w \Delta_{1} \Delta_{2}} \equiv l_{d}^{-2} .
$$

Equation 3.9 has an homogenous solution involving a linear combination of the modified Bessel functions $I_{0}(\sqrt{2} \kappa r)$ and $K_{0}(\sqrt{2} \kappa r)$. The lower boundary condition at $r=a$ is $\Phi=0$ (presuming the inner core radius is attached to a good conductor). The outer boundary condition at $r=b$ is that $D_{R}$ must be continuous

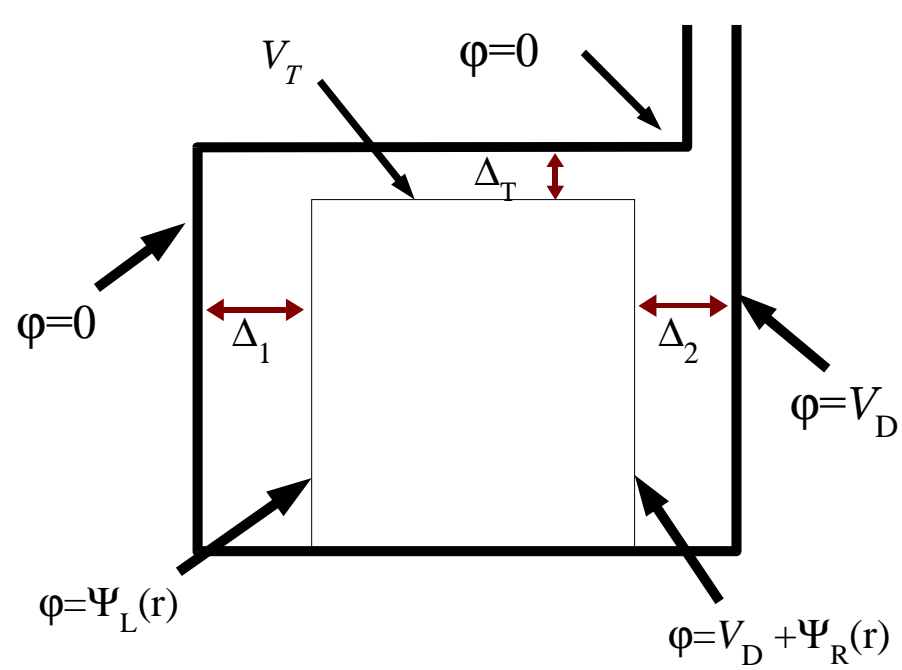

Fig. 4 - Illustrative cartoon showing the voltage configuration of a single core and the surrounding conducting surfaces. (i.e. the outer metallic band surrounding the core must have a net charge of zero).

The inhomogeneous solution for $\Phi$ is non-zero only if the RHS of Eq. 3.9 (which we now denote as $-S(r)$ ) is non-zero. The appropriate Green's function which satisfies the radial boundary conditions is 


$$
G\left(r, r^{\prime}\right)=r^{\prime}\left[I_{0}\left(\sqrt{2} \kappa r_{\triangleleft}\right)-K_{0}\left(\sqrt{2} \kappa r_{\triangleleft}\right) \frac{I_{0}(\sqrt{2} \kappa a)}{K_{0}(\sqrt{2} \kappa a)}\right] K_{0}\left(\sqrt{2} \kappa r_{\downarrow}\right)
$$

where $r_{\triangleleft}=\min \left(r, r^{\prime}\right)$ and $r_{\triangleright}=\max \left(r, r^{\prime}\right)$. Consequently.

$$
\Phi(r)=\int_{a}^{r} d r^{\prime} G\left(r, r^{\prime}\right) S\left(r^{\prime}\right)+C_{H}\left(\frac{I_{0}(\sqrt{2} \kappa r)}{I_{0}(\sqrt{2} \kappa a)}-\frac{K_{0}(\sqrt{2} \kappa r)}{K_{0}(\sqrt{2} \kappa a)}\right)
$$

If for illustrative purposes we presume that the Metglas core is placed symmetrically in $\mathrm{z}$ between the surrounding conducting plates with $\Delta_{1}=\Delta_{2} \equiv \Delta_{G}$, then $S(r) \equiv 0$ and $\kappa^{2}=\epsilon_{G} /\left(\epsilon_{R} w \Delta_{G}\right)$. Then we need only to satisfy the upper boundary condition on $D_{r}$ at $r=b$ by adding the appropriate homogenous solution. If the radial separation $\Delta_{T}$ between the outer edge of the core and the surrounding (conducting) metal enclosure is small compared to both $b$ and $w, D_{r} \approx \epsilon_{G}\left[V_{D} / 2+\Phi(b)\right] / \Delta_{T}$. We make further headway by realizing the effective decay length is quite short relative to the core radial dimensions for the DARHT-2 cell:

$$
l_{d} / \sqrt{2}=\left[\frac{(3.2 /(1-0.75))}{2.3} \frac{4 \times 0.25}{2}\right]^{1 / 2} \approx 1.7 \text { inches }
$$

where we have presumed a packing fraction $p_{f}=0.75$, a dielectric constant for oil $\epsilon_{G}=2.3$, a dielectric constant for mylar $\epsilon_{I}=3.2$ and a gap spacing $\Delta_{G}=0.25$ inches. Consequently, we may use the large argument approximation for the Bessel functions with $I_{0}(x) \approx e^{x} / \sqrt{2 \pi x}$ and $K_{0}(x) \approx e^{-x} \sqrt{\pi / 2 x}$. Thus, $D_{R}($ interior $) \approx-\sqrt{2} \epsilon_{R} \kappa \Phi(b)$. Solving for $\Phi(b)$ we find

$$
\Phi(b) \approx-\frac{V_{D}}{2} \times\left(1+\frac{\sqrt{2} \epsilon_{R} \Delta_{T}}{\epsilon_{G} l_{d}}\right)^{-1} \text { and } V_{T} \approx \frac{V_{D}}{2} \times\left(1+\frac{\epsilon_{G} l_{d}}{\sqrt{2} \epsilon_{R} \Delta_{T}}\right)^{-1}
$$

For large $\Delta_{T} / l_{d}$, the potential at the top of the Metglas approaches the "normal" value of $V_{D} / 2$, i.e. exactly halfway between the drive and ground potential. For $\Delta_{T} \ll l_{d}$, the potential at the top of the core $V_{T} \sim \sqrt{2} V_{D} \Delta_{T} / l_{d}$ and is thus much closer to ground potential (due to the large capacitance between the outer band and the surrounding metal can). Numerically for DARHT-2, $\Delta_{T} \approx 1.0$ inches, $\sqrt{2} \epsilon_{R} \Delta_{T} / \epsilon_{G} l_{d} \approx 3.3$, and we predict $V_{T} \approx 0.38 V_{D}$. The predicted longitudinal electric field stress (for this single core example) is $\sim 60 \%$ higher at outer right edge of the core as compared with the outer left region.

Due to the exponential dependence of $\Phi$ with $r$, in a distance of less than 10 inches from the outer radius it drops by more than a factor of 100 and the longitudinal electric field stress at the left and right sides of the core are nearly equal (again presuming $\Delta_{1}=\Delta_{2}$ ). As one will see from later examples, this rapid convergence with decreasing $r$ to equal 
longitudinal electric field stress division is a general property of the core voltage solution so long as $\kappa(b-a) \gg 1$. This has the immediate consequence that any enhanced breakdown problems are far more likely to occur near the outer few inches of the core than in the inner radial regions.

\section{Physical Interpretations and Equivalent Circuits}

The analysis in Section 3 shows that, in the case of a single core with equal gap spacings, the only source for the electrostatic potential $\Phi(r)$ inside the core region is the free charge on the inside of the outer band ( $\equiv q$ ). Since in normal operation the outer band is "floating", the net free charge on it is zero; the charge on the outside of the outer band must equal $-q$.

A physical interpretation of the exponentially decaying character of the homogeneous solution for $\Phi(r)$ in Eq. (3.9) can be obtained from an analysis of the distributed circuit model shown in Fig. 5. If we consider a layer of the core between $r$ and $r+d r$ in Fig. 5, the

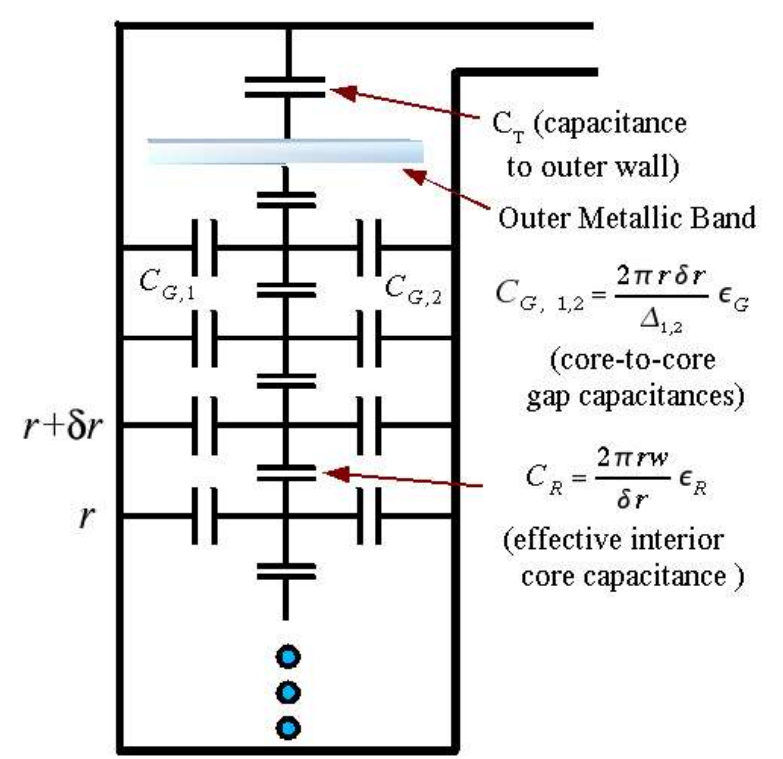

Fig. 5-Distributed circuit model for the electrostatic component of the cell voltage net radial displacement current passing through the core at this radius $r$ is given by

$$
I_{\text {displacement }}=-\frac{2 \pi r w \epsilon_{R}}{\delta r} \frac{\partial}{\partial t}[\Phi(r+\delta r)-\Phi(r)]
$$

This defines an effective "core capacitance" $C_{R}$ of that layer as given in Fig. 5. The radial displacement current through the core decreases as we move inward since it is "shunted out" each side through the gap capacitances at each layer given by $C_{G 1,2}$ in Fig. 5. If we write down the differential equation for the electrostatic voltage $\Phi(r)$ that follows from this distributed circuit model, we obtain the homogeneous version of Eq. (3.9).

The approximate solution for the electrostatic voltage when $l_{1} \ll b-a$ with $l_{1} \equiv l_{d} / \sqrt{2}$ is

$$
\Phi(r) \approx \Phi(b) \exp -(b-r) / l_{1}
$$

Since the total electrostatic voltage drop from the outer band to ground is $\Phi(b)$, it makes sense to define an "effective core-gap capacitance" as

$$
q=C_{e f f} \Phi(b)
$$


Relating the total charge $q$ on the inside of the outer band to $D_{r}(b)=-\varepsilon_{R} \Phi(b) / l_{1}$, we have

$$
C_{e f f}=2 \pi \epsilon_{R} \frac{b w}{l_{1}}
$$

Note that for the case of with no applied inductive voltage, we could set up an electrostatic potential of the same form as Eq. (4.2) inside the core by connecting a pulsed (or AC) generator between the outer band on the core and ground. Indeed, for $\Delta_{T} \rightarrow \infty$, measurement of the $\mathrm{AC}$ current flowing into the outer band from this generator provides a direct measurement of $C_{\text {eff }}$. This measurement has recently been made on the LBNL small core test stand and will be reported elsewhere.

With an inductive voltage applied and equal core-to-core gap spacings, the voltage on the outer band is

$$
V_{T}=\frac{V_{D}}{2}+\Phi(b)
$$

The equivalent lumped circuit model shown in Fig. 6 can therefore be used to calculate the voltage on the outer band, where

$$
C_{T}=\varepsilon_{T} \frac{2 \pi b w}{\Delta_{T}}
$$

is defined as the capacitance of the top band to the outer metal cylinder shown in Fig. 4. From this circuit we have

$$
V_{T}=\frac{V_{D} / 2}{1+C_{T} / C_{e f f}}
$$

in agreement with Eq. (3.13). This illustrates the physical point that the deviation from a simple equal division of inductive voltage across the two gaps is due to the capacitance of the outer band to the outer wall at ground potential. It also shows that it is the ratio of this capacitance to an "effective core-gap" capacitance that enters into the voltage distribution. We note that a similar capacitive model was previously employed by L. Schlitt et al. (see PSI-FR-243-3) in work modeling the voltage division in the Hermes-III accelerator cell.

The same physical concepts will clearly be involved in more general cases. As one generalization that provides further insight, consider a single core cell with unequal gap spacings. In addition to the homogeneous solution for $\Phi$ from Eq. (3.9), we now need a 
particular solution $\Phi_{p}(r)$. If the scale length for radial variations in $V(r)$ are long compared to $l_{1}$, an approximate solution for $\Phi_{p}(r)$ is

$$
\Phi_{p}(r) \approx\left(\frac{\Delta_{1}-\Delta_{2}}{\Delta_{1}+\Delta_{2}}\right) \frac{V(r)}{2}
$$

As before, the homogeneous solution in Eq. (4.2) will be a good approximation as long as $l_{1} \ll b-a$. Several $l_{1}$ scale lengths inward from the top band, therefore, the left and right "full voltages" will contain only $\Phi_{p}(r)$ and $V(r)$. From Eqs. (3.4a) and (3.4b), using the above approximation, we find that the electric field stress in both gaps is the same in this region well inside the outer band,

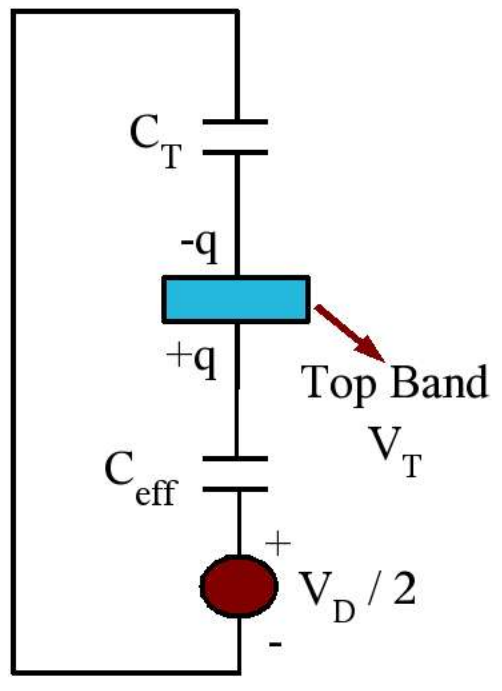

Fig. 6. Lumped circuit model for the voltage distribution

$$
E_{z, L}(r) \approx E_{z, R}(r) \approx-\frac{V(r)}{\Delta_{1}+\Delta_{2}}
$$

In fact, inspection of Eq. (3.6) shows that this equipartition of stress is a general result (applicable even to multi-core cases as we discuss later in Sections 5 and 6) whenever the radial variation of $\Phi$ is slow compared to the scale length $l_{d}$ (or its appropriate generalization in multi-core cases).

Near the top band, the gradient in $\Phi(r)$ will be dominated by the homogenous solution, so the physical arguments given above that led to the identification of $C_{\text {eff }}$ remain valid. We find that with unequal gaps the lumped circuit model in Fig. 6 and the expression for the voltage on the outer band in Eq. (4.7) are the same except for the replacement of $V_{D} / 2$ by the quantity $\left(\frac{\Delta_{1}}{\Delta_{1}+\Delta_{2}}\right) V_{D}$.

\section{Two Core Solution}

In going from one to two cores, we must introduce some new notation. Let $V_{1}$ and $V_{2}$ be the inductive voltages of cores \#1 and \#2, respectively, with the total inductive voltage being $V_{D}=V_{1}+V_{2}$. Similarly $\Phi_{1}$ and $\Phi_{2}$ will be the $z$-independent electrostatic potential in the two cores. Let $\Delta_{2}$ now be the longitudinal separation between the two cores and $\Delta_{3}$ be the separation between core \#2 and the drive plane. We also define the longitudinal electric field in the gaps between the cores: 


$$
\begin{aligned}
& \Delta_{1} E_{z, 1}=-\left(\frac{V_{1}(r)}{2}+\Phi_{1}(r)\right) \\
& \Delta_{2} E_{z, 2}=-\left(\frac{V_{2}(r)-V_{1}(r)}{2}+\Phi_{2}(r)-\Phi_{1}(r)\right) \\
& \Delta_{3} E_{z, 3}=-\frac{V_{2}(r)}{2}+\Phi_{2}(r)
\end{aligned}
$$

Following the same mathematical development as in the single core case for relating the radial dependence of $\Phi$ in each core to the jump in $D_{z}$ from side of the core to the other, one may use Eq. (3.6) to show that there are two natural eigenvectors: the sum $\Phi_{1}+\Phi_{2}$ and the difference $\Phi_{1}-\Phi_{2}$. The former is related to the sum $E_{z, 1}+E_{z, 3}$ :

$$
\frac{1}{r} \frac{\partial}{\partial r} r \frac{\partial\left(\Phi_{1}+\Phi_{2}\right)}{\partial r}-\bar{\kappa}^{2}\left(\Phi_{1}+\Phi_{2}\right)=\bar{\kappa}^{2}\left\{\frac{\Delta_{3} V_{1}(r)-\Delta_{1} V_{2}(r)}{2 \bar{\Delta}}+\frac{\Delta_{d}\left(\Phi_{1}(r)-\Phi_{2}(r)\right)}{\bar{\Delta}}\right\}
$$

where $\bar{\kappa}^{2} \equiv \frac{\epsilon_{G}}{\epsilon_{R}} \times \frac{\bar{\Delta}}{w \Delta_{1} \Delta_{3}}, \quad 2 \bar{\Delta} \equiv \Delta_{1}+\Delta_{3} \quad$ and $\quad 2 \Delta_{d} \equiv \Delta_{3}-\Delta_{1}$. The latter similarly involves the "three-point" electric field operator $-2 E_{z, 2}+E_{z, 1}+E_{z, 3}$. However, the resulting equation is rather messy (and uninformative) unless we make the assumption of equal gap spacing between the cores: $\Delta_{1}=\Delta_{2}=\Delta_{3} \equiv \Delta_{G}$. Then we find

$$
\frac{1}{r} \frac{\partial}{\partial r} r \frac{\partial\left(\Phi_{1}-\Phi_{2}\right)}{\partial r}-3 \bar{\kappa}^{2}\left(\Phi_{1}-\Phi_{2}\right)=-\bar{\kappa}^{2}\left\{\frac{V_{1}(r)+V_{2}(r)}{2}\right\}
$$

indicating that the eigenvalues for the sum and difference eigenvectors are $\bar{\kappa}$ and $\sqrt{3} \bar{\kappa}$ respectively.

At radii $r$ which are a couple decay lengths or more smaller than $b$, one may neglect the radial derivative term on the LHS of Eq. (5.2) to find the (inhomogeneous) solution

$$
\Phi_{1}-\Phi_{2} \approx\left[V_{1}(r)+V_{2}(r)\right] / 6
$$

Then after applying Eq. (3.14), we obtain $\Phi_{1}(r)=-\Phi_{2}(r)=\left[V_{1}(r)+V_{2}(r)\right] / 12$. For $V_{1}(r)=V_{2}(r) \equiv V_{D}(r) / 2$, the electric fields in each of the three gaps have the identical value

$$
E_{z, 1}(r)=E_{z, 2}(r)=E_{z, 3}(r)=-\frac{V_{D}(r)}{3 \Delta_{G}}
$$


indicating an "equipartition" of the total (inductive + electrostatic) voltage between the two cores.

To satisfy the boundary condition for a continuous $D_{R}$ at the top of each core, we must add an additional homogenous solution $\Phi_{H}$ to each of the inhomogeneous solutions. However, we defer this calculation until the next section.

\section{General Multicore Solution}

For the general case of multiple cores (i.e. $N>1$ ), we presume each core $n$ has an inductive voltage $V_{n}$ together with an electrostatic voltage $\Phi_{n}$ which satisfy the appropriate boundary conditions. To simplify the analysis, let us presume that all of the longitudinal gaps spacings between adjacent cores have the identical value of $\Delta_{G}$. For each individual core, we then have

$$
\frac{1}{r} \frac{\partial}{\partial r} r \frac{\partial \Phi_{n}}{\partial r}=\kappa^{2}\left\{2 \Phi_{n}-\Phi_{n-1}-\Phi_{n+1}+\frac{1}{2} V_{n-1}(r)-\frac{1}{2} V_{n+1}(r)\right\}
$$

with $\kappa$ defined as before and $V_{0} \equiv 0$ and $V_{N+1} \equiv 0$.

To solve the coupled problem, we note that the first three terms on the RHS of the above equation be rewritten as a tridiagonal matrix (which we denote as $M$ ):

$$
l_{d}^{2} \nabla_{r}^{2}\left[\begin{array}{c}
\Phi_{1} \\
\Phi_{2} \\
\Phi_{3} \\
\vdots \\
\Phi_{N-2} \\
\Phi_{N-1} \\
\Phi_{N}
\end{array}\right]=\left[\begin{array}{cccccccc}
2 & -1 & 0 & 0 & \cdots & 0 & 0 & 0 \\
-1 & 2 & -1 & 0 & \cdots & 0 & 0 & 0 \\
0 & -1 & 2 & -1 & 0 & \cdots & 0 & 0 \\
\vdots & \vdots & \vdots & \vdots & \vdots & \vdots & \vdots & \vdots \\
0 & 0 & \cdots & 0 & -1 & 2 & -1 & 0 \\
0 & 0 & \cdots & \cdots & 0 & -1 & 2 & -1 \\
0 & 0 & \cdots & \cdots & 0 & 0 & -1 & 2
\end{array}\right]\left[\begin{array}{c}
\Phi_{1} \\
\Phi_{2} \\
\Phi_{3} \\
\vdots \\
\Phi_{N-2} \\
\Phi_{N-1} \\
\Phi_{N}
\end{array}\right]+\frac{1}{2}\left[\begin{array}{c}
-V_{2} \\
V_{1}-V_{3} \\
V_{2}-V_{4} \\
\vdots \\
V_{N-3}-V_{N-1} \\
V_{N-2}-V_{N} \\
V_{N-1}
\end{array}\right]
$$

with each $\Phi$ and $V$ being functions of $r$.

We need to determine the eigenvectors $p^{(i)} \equiv\left(p_{1}^{(i)}, p_{2}^{(i)}, \cdots p_{N-1}^{(i)}, p_{N}^{(i)}\right)$ and eigenvalues $\alpha_{(i)}$ which satisfy $\operatorname{det}\left(M-\alpha^{2}\right)=0$ and $\left(M-\alpha_{(i)}^{2}\right) \cdot p^{(i)}=0$. Here $p_{j}^{(i)}$ is defined as the value of the voltage on cell $j$ corresponding to the $i^{\text {th }}$ eigenvector. For $N=1$, there is one eigenvalue $\alpha^{2}=2$. For $N=2$, the requirement that the determinant $\left(2-\alpha^{2}\right)^{2}-1 \equiv D_{2}$ equal zero results in eigenvalues of 1 and 3 for $\alpha^{2}$, agreeing with what we found in the last section. The corresponding (orthonormal) eigenvectors are $\left(\frac{1}{\sqrt{2}}, \frac{1}{\sqrt{2}}\right)$ and $\left(\frac{1}{\sqrt{2}}, \frac{-1}{\sqrt{2}}\right)$. For $N=3, \operatorname{det}\left(M-\alpha^{2}\right)=\left(2-\alpha^{2}\right)\left[\left(2-\alpha^{2}\right)^{2}-2\right] \equiv D_{3}$ results in three 
eigenvalues $(2-\sqrt{2}, 2,2+\sqrt{2})$ for $\alpha^{2}$. The corresponding orthonormal eigenvectors are:

$$
\left(\frac{1}{2}, \frac{1}{\sqrt{2}}, \frac{1}{2}\right),\left(\frac{1}{\sqrt{2}}, 0,-\frac{1}{\sqrt{2}}\right),\left(\frac{1}{2},-\frac{1}{\sqrt{2}}, \frac{1}{2}\right)
$$

To determine the eigenvalues for larger $N$, one can use the recursion relation for the determinants

$$
D_{N}=\left(2-\alpha^{2}\right) D_{N-1}-D_{N-2}
$$

For $N$ odd, one can see that $\alpha^{2}=2$ must always be one of the eigenvalues.

Once the eigenvalues are found, the individual components of the (unnormalized) eigenvectors may be found from the recursion relation:

$$
p_{1}^{(i)}=1 \quad p_{2}^{(i)}=2-\alpha_{(i)}^{2} \quad p_{3}^{(i)}=\left(2-\alpha_{(i)}^{2}\right)^{2}-1 \quad \cdots \quad p_{J}^{(i)}=D_{J-1}\left(\alpha_{(i)}^{2}\right)
$$

The appropriate normalization constant may be found by taking the norm of the unnormalized eigenvector. Corresponding to each eigenvalue will be an inhomogeneous solution $\Phi_{I}^{(i)}(r)$ with a source function $S_{I}^{(i)}(r)=-p^{(i)} \cdot \vec{S}(r)$ where $\vec{S}(r)$ is the last term on the RHS of Eq. 3.19. This source will be used with the Green's function given in Eq. 3.10 with the factor $\sqrt{2} \kappa$ being replaced by $\alpha_{(i)} \kappa$. For $S_{I}^{(i)}(r)$ slowly varying on the length scale $l_{d}, \alpha_{(i)} \kappa r \gg 1$ and $\alpha_{(i)} \kappa(b-r) \geqslant 2$, one finds $\Phi_{I}^{(i)}(r) \approx S^{(i)}(r) / \alpha_{(i)}^{2}$. However, as $r$ approaches $b$ (i.e. the top of the core), $\Phi_{I}^{(i)}(r) \rightarrow S^{(i)}(b) / 2 \alpha_{(i)}^{2}$, the reduction by a factor of two being attributable to the fact that the source term is zero for $r>b$.

To finish the solution for $\Phi$, we must also determine the homogenous solutions $\Phi_{H}^{(i)}$ which must be added to $\Phi_{I}^{(i)}$ satisfy the requirement that the inner and outer solutions for displacement field $D_{R}$ be matched at $r=b$ on an eigenvector by eigenvector basis. Consequently,

$$
\frac{\epsilon_{G}}{\Delta_{T}}\left(V^{(i)}(b)+\Phi_{I}^{(i)}(b)+\Phi_{H}^{(i)}(b)\right)=-\left.\epsilon_{R} \frac{\partial}{\partial r}\left(\Phi_{I}^{(i)}(r)+\Phi_{H}^{(i)}(r)\right)\right|_{r=b}
$$

where $V^{(i)}(b) \equiv p^{(i)} \cdot \vec{V}_{t}$ and $V_{t,(n)}=\sum_{j=1}^{n-1} V_{j}(b)+\frac{1}{2} V_{n}(b)$ represents a "net" inductive voltage at the top of the $n^{\text {th }}$ core. For $\alpha_{(i)} b \gg l_{d}, \partial \Phi_{H} / \partial r \approx \alpha_{(i)} \Phi_{H}^{(i)} / l_{d}$ and $\partial \Phi_{I} / \partial r \approx-\alpha_{(i)} \Phi_{I}^{(i)} / l_{d}$, again presuming that the source term for the inhomogeneous solution is varying on a length scale much greater than $l_{d} / \alpha_{(i)}$. The solution of Eq. 6.5 then gives 


$$
\begin{gathered}
\Phi_{H}^{(i)}(b)=-\frac{\left(V^{(i)}(b)+\left(1-\frac{\epsilon_{R} \alpha_{(i)} \Delta_{T}}{\epsilon_{G} l_{d}}\right) \Phi_{I}^{(i)}(b)\right)}{1+\frac{\epsilon_{R} \alpha_{(i)} \Delta_{T}}{\epsilon_{G} l_{d}}} \\
V_{T}^{(n)}=-\sum_{i} p_{n}^{(i)} \times\left(\frac{V^{(i)}(b)+2 \Phi_{I}^{(i)}(b)}{1+\frac{\epsilon_{G} l_{d}}{\epsilon_{R} \alpha_{(i)} \Delta_{T}}}\right)
\end{gathered}
$$

As before, $V_{T}^{(n)} \rightarrow 0$ in the limit $\Delta_{T} \rightarrow 0$ (i.e., infinite capacitance to the ground plane). For $\Delta_{T} \gg \epsilon_{G} l_{d} / \epsilon_{R}$, the total voltage at the top of core $n$ has an asymptotic value of $V_{t,(n)}+2 \Phi_{I}(b)$. Defining $\zeta \equiv \epsilon_{G} l_{d} / \epsilon_{R} \Delta_{T}$, we numerically computed the voltages at the outer radius of the individual cores for 3-and 4-core systems as functions of $\zeta$ presuming that both $a$ and $b$ are much greater than $l_{d}$. Figures 7 and 8 display the ratio $V_{T}^{(n)}(\zeta) / V_{D}$ which shows $V_{T}^{(n)} \rightarrow n /(N+1)$ as $\zeta \rightarrow 0$. For $\zeta \geq 1$, the top voltage can drop to less than half its value at $\zeta=0$.

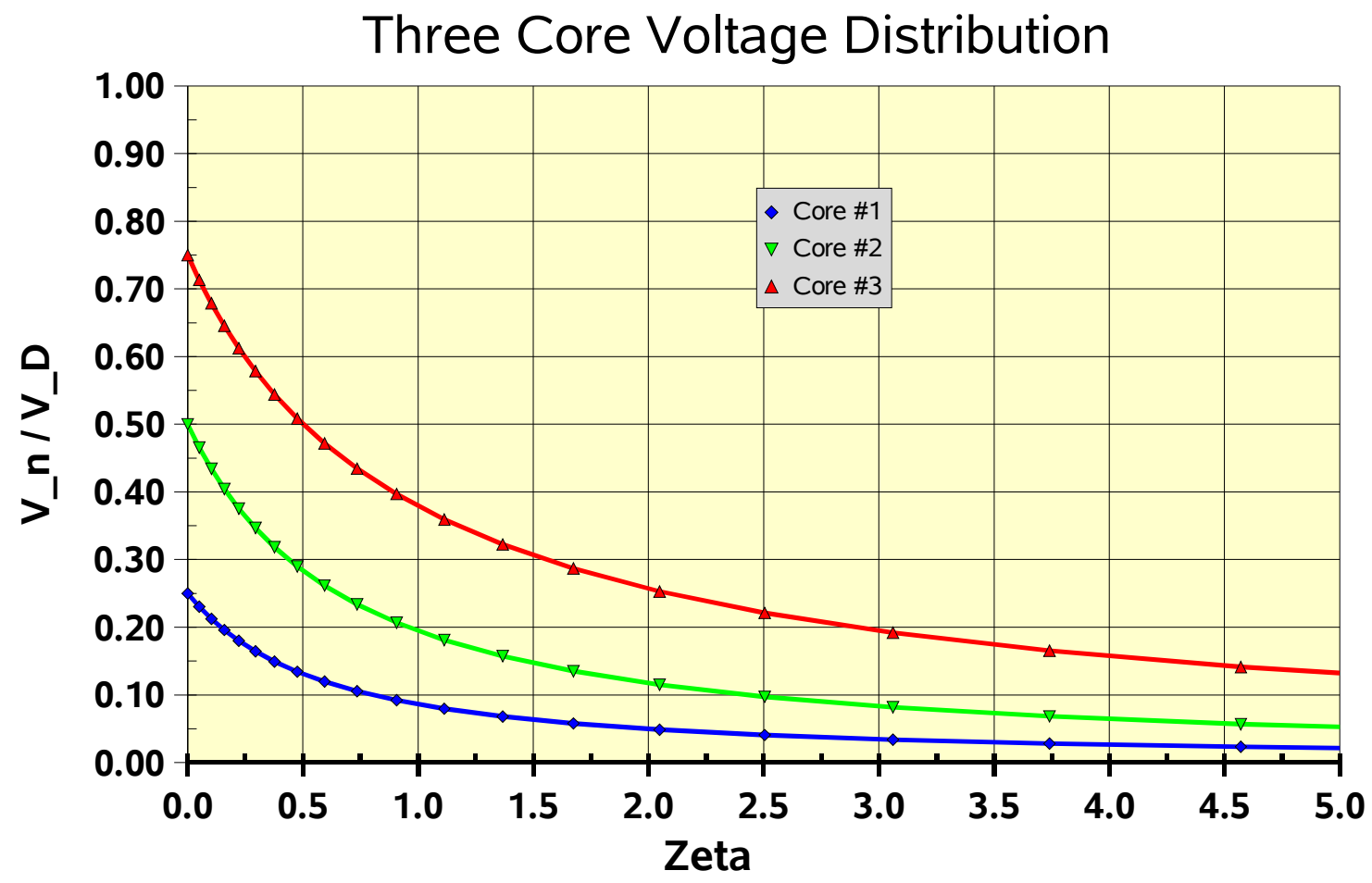

Fig. 7 - Normalized voltages at the outer radius of individual cores in a 3-core cell 


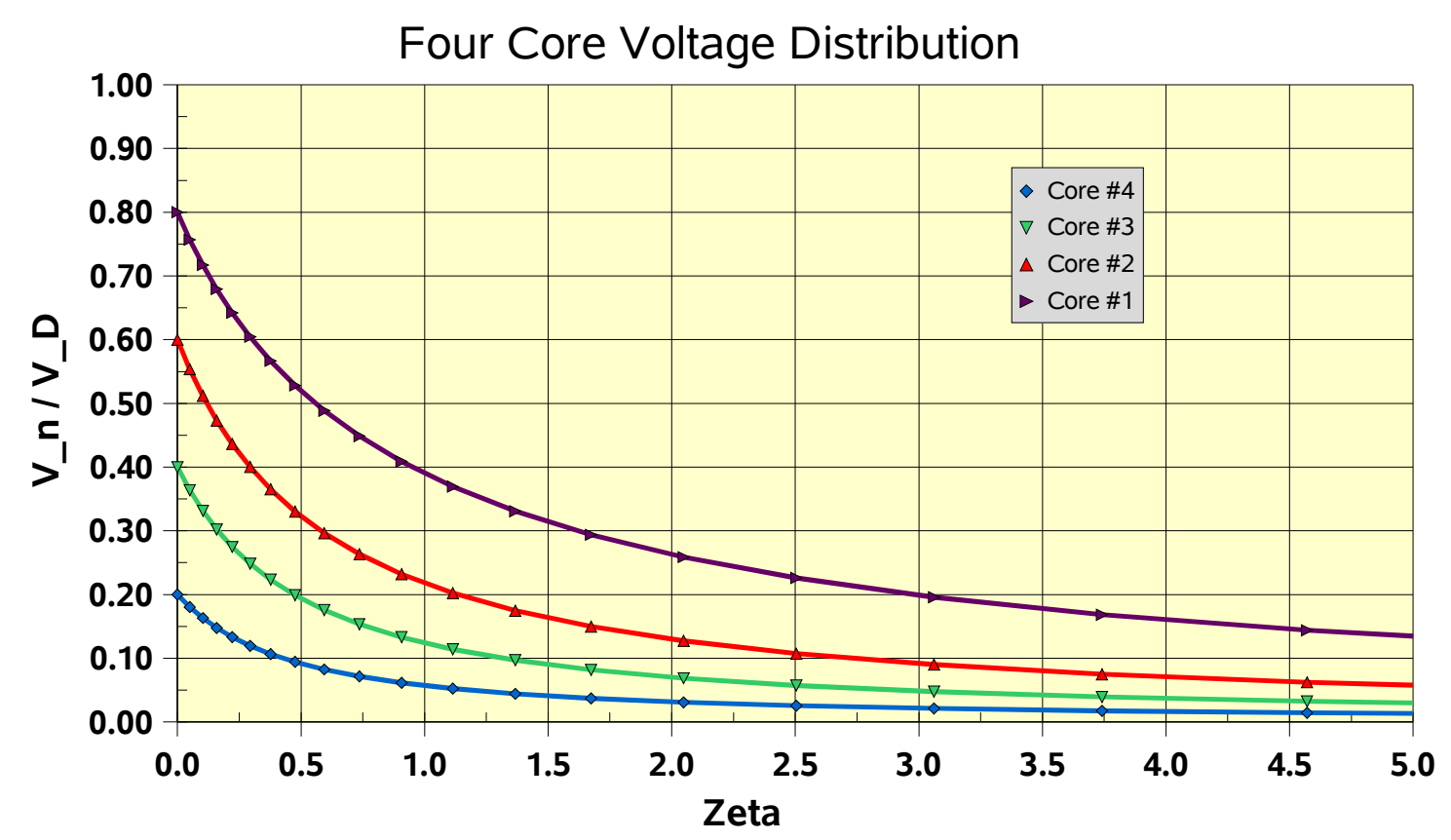

Fig. 8 Normalized voltages at the outer radius of individual cores in a 4-coreaccelerator cell

Presuming that $V_{n}(r)$ slowly varies with $r$ relative to $l_{d} \times \max \left(\alpha_{(i)}^{-1}\right)$, deep in the core the radial derivative term on the LHS of Eq. 3.20 is very small. This smallness forces $E_{z}$ in the gaps to the left and right of each core to have a nearly equal value. This can only be true if and only if $E_{z}$ has a constant value $-V_{D} /\left[(N+1) \Delta_{G}\right]$ in all the gaps. We believe that this is a general property of core geometries like DARHT-2 accelerator cell (see Fig. 9) for which $(b-a) \gg \sqrt{w \Delta_{G}}$ applies, even when the values for individual core voltseconds and/or the gap separations vary significantly. In other words, the deviation from equipartition of voltages in a multicore geometry is strongest at the outer radial boundary and becomes very small a few $l_{d}$ skin depths in the radial interior.

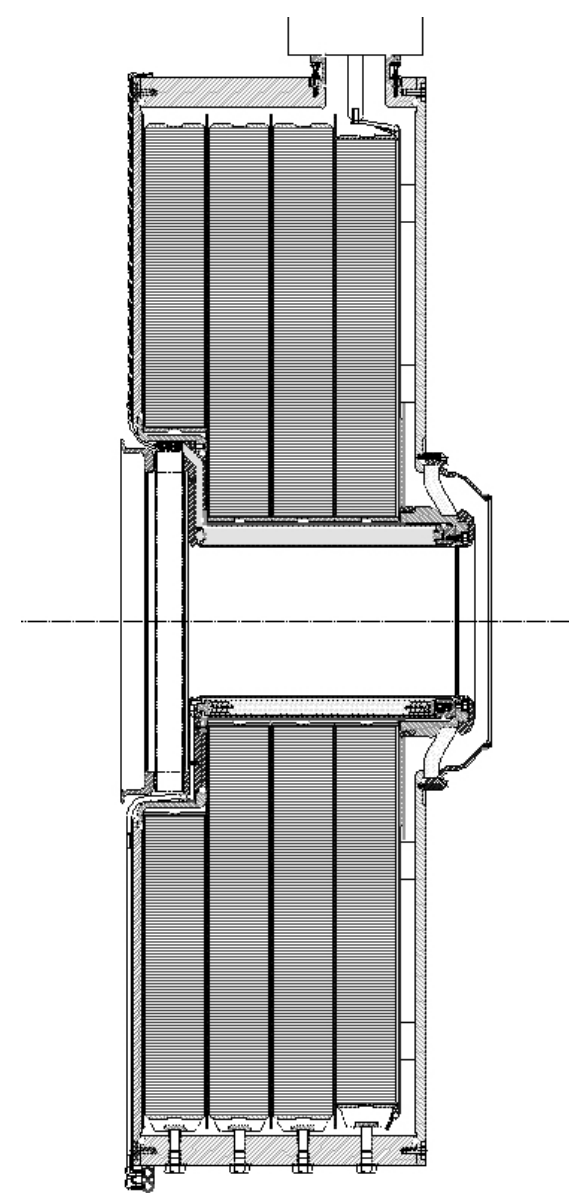

Fig. 9 - Side view of geometry of a 4-core DARHT-2 accelerator cell 



Fig. 10-12-Contours of $4^{\text {th }}$ core voltage normalized to $V_{D}$ at its outer core boundary for $(b / a)=1.2,1.5$, and 3.0 (moving in a clockwise direction from above to the right) plotted versus $\zeta$ and $a / l_{d}$. The contour levels are 0.75, 0.65, 0.55, 0.45, 0.35 and 0.25 moving from the lower left toward the upper right.

\section{More Generalized Solution for}

$$
V_{\text {TOT }}(a, b, \zeta)
$$

In the more general case where the ratio of $(b / a)$ may be large and $\left(a / l_{d}\right)$ is not necessarily large, one must use Eqs. (3.11) and (6.7) together with $V_{N}(r)$ varying as

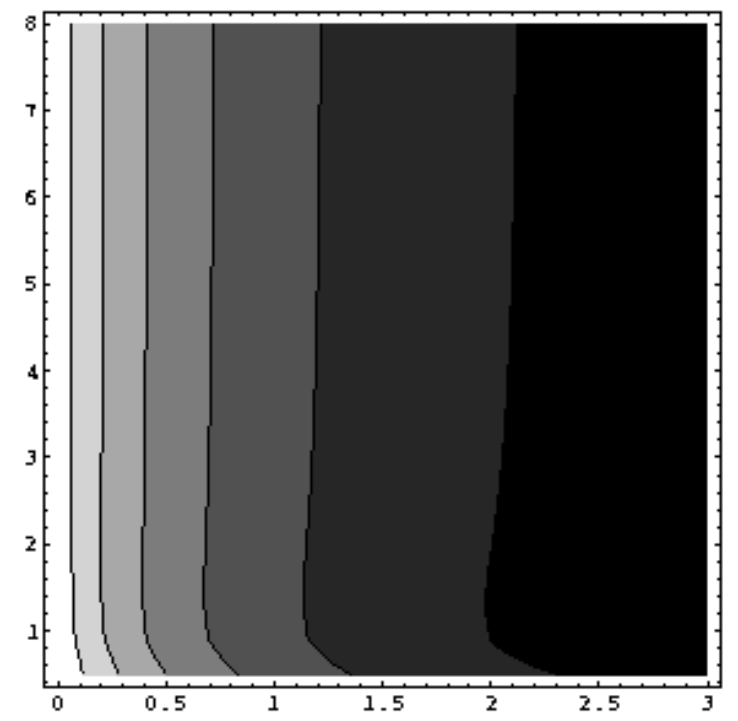
$\log (r / a)$ to determine the inhomogeneous and homogenous solutions for the individual cores. In general, this is merely a question of some straight-forward algebra and integration, although the needed indefinite integral (for $x$ positive)

$$
\int d x \times I_{0}(x) \ln x=x I_{1}(x) \ln x-I_{0}(x)
$$

is surprisingly not available in "standard" integral compendia such as Gradshteyn and Ryzhik. Presuming that the inner radial core boundary $(r=a)$ is at zero potential and the cores and gaps between them have identical dimensions, one can solve for $\Phi_{I}^{(i)}(r)$ and $\Phi_{H}^{(i)}(r)$. In Figs. 10-12 (ordered in a clockwise direction) we present the results for the total voltage at the outer core radius for core \#4 in a 4-core system as functions of $\left(a / l_{d}, \zeta\right)$ with $\zeta \equiv \epsilon_{G} l_{d} / \epsilon_{R} \Delta_{T}$ for different ratios of $(b / a)$. One sees that the voltage rapidly drops from the asymptotic value of 0.8 as $\zeta$ increases and that the sensitivity to $\zeta$ increases at smaller $(b / a)$ ratios. 


\section{Some Examples Relevant to the DARHT-2 Project}

One can use the analytical framework developed above to examine some specific examples of interest. The first is the multicore test stand at LBNL for which individual core voltage measurements were made in the November 2003 - February 2004 time period. The test stand included up to four identical Metglas cores within a conducting metal can enclosure. The cores had 4-inch widths together with inner diameters of 8.0 inches and outer diameters of 14.0 inches. Hence, the ratio $b / a=1.75$ lies in the parameter region in which the variation of $H_{\theta}$ with $r$ is moderately large. Acrylic spacers with an effective $\epsilon_{G}=1.75$ of varying thickness were used between the individual cores. Taking into account the Mylar tape between each winding, the effective interior core dielectric is $\epsilon_{R} \approx 8.3$. With air separating the surrounding conducting cylinder from the outer radial boundary of the cores, $\epsilon_{T}=1$. Given these parameters, from the analysis of the preceding sections, one predicts $l_{d} \approx 1.7$ inches and $\left(a / l_{d}\right) \approx 1$.

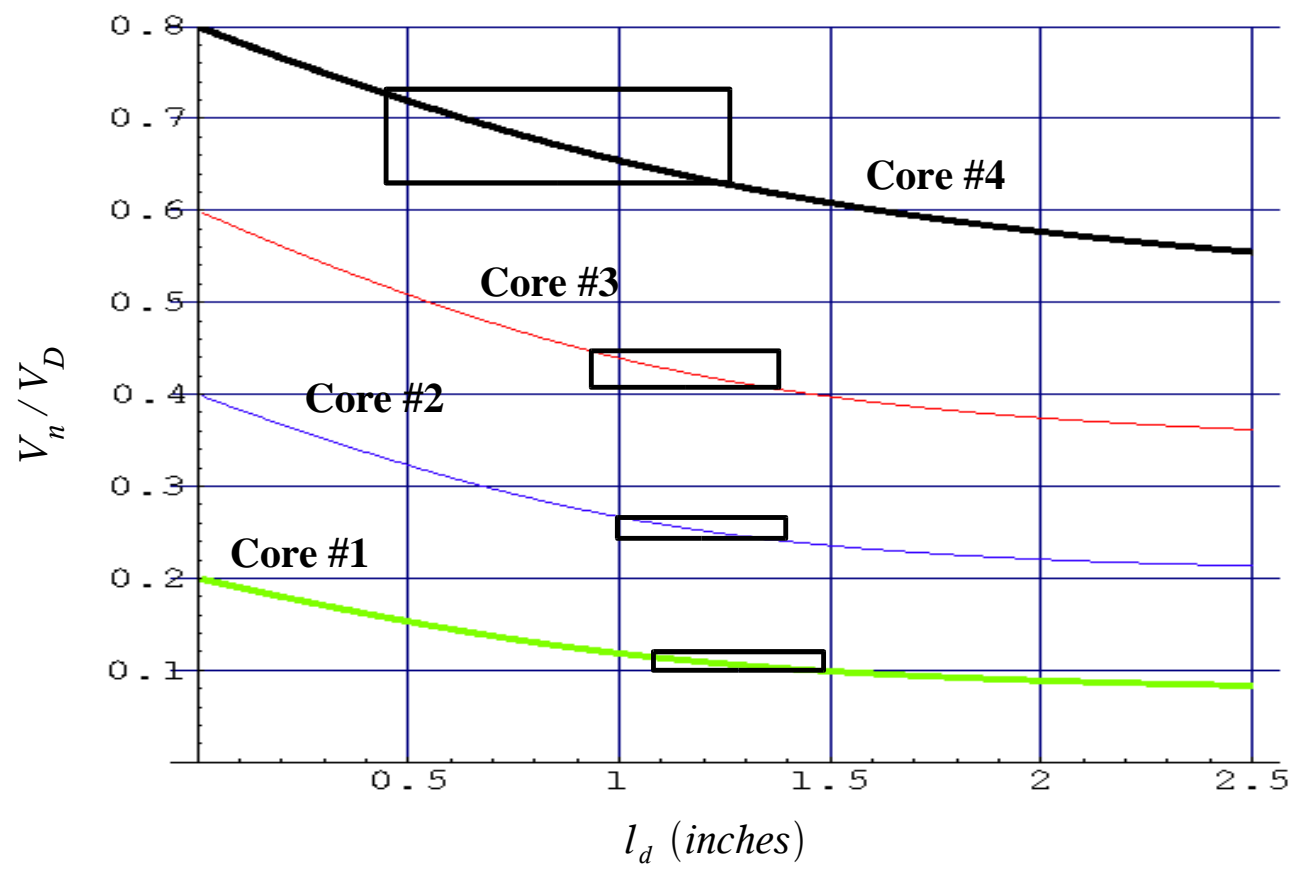

Fig. 13 - Predicted voltages in a 4-core system normalized to the drive voltage as a function of the decay length $l_{d}$ for the core parameters corresponding to the LBNL 4-core test stand. The black open boxes refer to voltage data taken by L. Reginato and W. Waldron.

Figure 13 shows the predicted ratios for $V_{n} / V_{D}$ as a function of $l_{d}$ for $n=1-4$ and $\Delta_{T}=1.0$ inches. Superimposed on the separate curves are boxes representing voltage data at the outer core radius taken by L. Reginato and W. Waldron of LBNL in December 2004 . From this data, it appears that the best fit would imply that the decay length is somewhere between 1.1 and 1.3 inches, nearly 30\% smaller than the value expected from a "first principles" application of our analysis. 


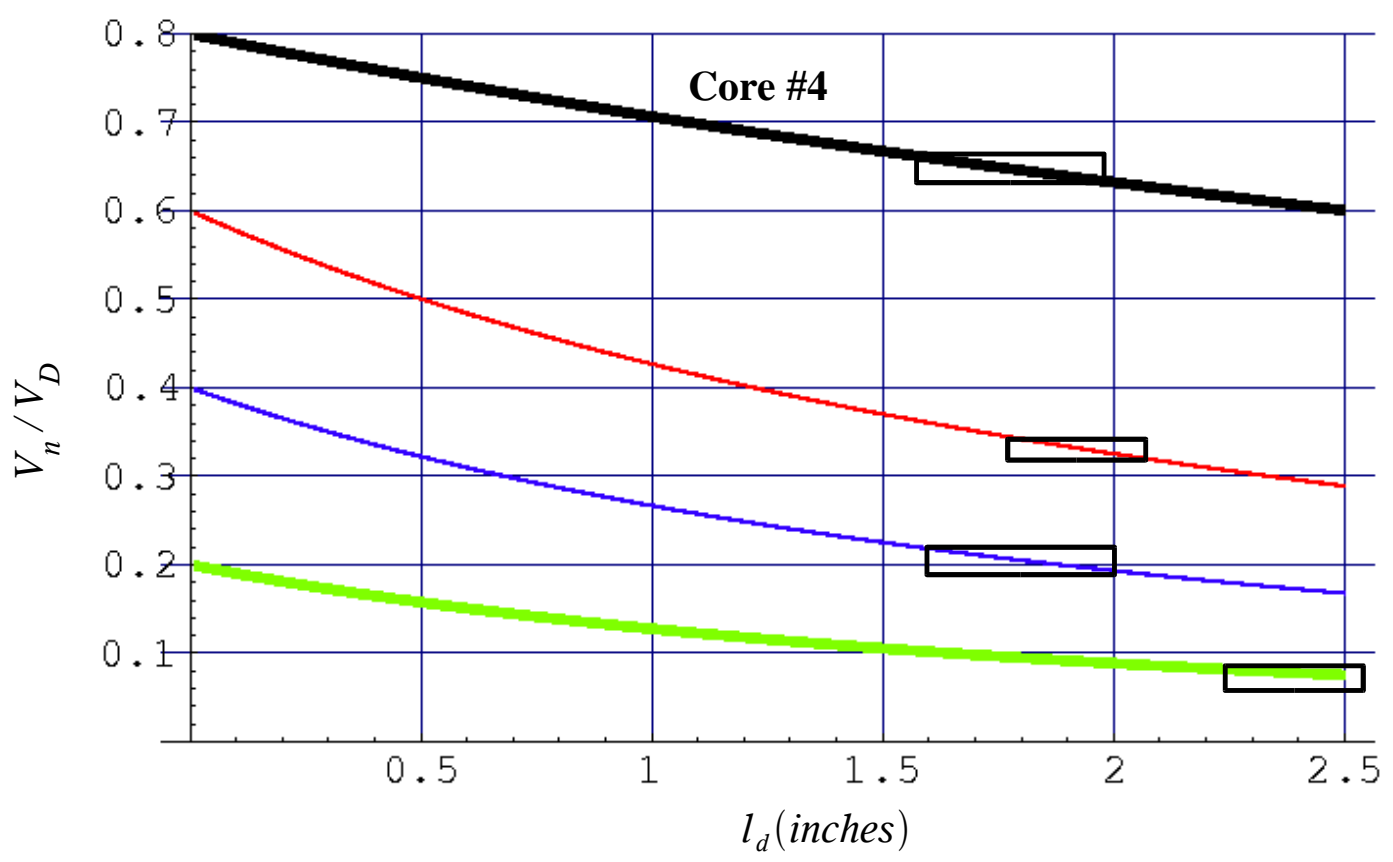

Fig. 14 - Predicted core voltages normalized to the drive voltage at the outer radius for a DARHT-2 accelerator cell as a function of decay length $l_{d}$. For cores \#2-\#4 the inner radius a was set to 8.0" while for core \#1 it was 13.0". For all cores the outer radius was set to 33.0”. For core \#4, $\Delta_{T}$ was twice as great for the other cores. The black open boxes indicate results for $V_{n} / V_{D}$ from LSP code results obtained by T. Hughes of MRC.

In Fig. 14 we show the predicted core voltages at their outer radius as a function of the decay length $l_{d}$ for a 4-core accelerator cell whose geometry is that of the DARHT-2 standard cell. For each individual core, we determined its voltage solution by presuming that all four cores had the same values of of $a, b$, and $\Delta_{T}$. Although this is not physically consistent, it gives a first order correction to otherwise presuming that the average value of these quantities applied to each and every core. However, the calculation did presume equal core-to-core gap spacing which is not true in reality (without this assumption, the solution for the eigenvalues and eigenfunctions becomes very ugly). The open boxes show the ratios of $V_{n} / V_{D}$ as calculated by T. Hughes of MRC using the LSP code; these results were presented at the October 2003 DARHT-2 TriLab meeting at LANL. While these quantitative results are not expected to show agreement at the percent level, we note that the predicted $l_{d}$ lies in the range 1.7-2.0". This is about $25 \%$ less than that predicted by first principles (see Eq. 3.12) presuming a core-to-core longitudinal spacing of 0.25 inches. Although the actual core-to-core spacing varies by $30 \%$ or more, the square-root dependence of $l_{d}$ upon $\Delta_{n}$ make it unlikely that this variation alone is responsible for difference between predicted analytical and LSP-simulated values of $V_{n} / V_{D}$. 


\section{Conclusions}

In this note we have shown that a simple microscopic model of core winding magnetization can be used to help determine a macroscopic solution of the overall inductive and electrostatic voltage distribution in a multiple core induction cell. We find that when the core-to-outer-enclosure capacitance becomes large relative to the core-tocore capacitance, the voltage in the outer radial regions of individual cores can deviate strongly away that expected from a simple uniform distribution of voltages amongst the cores. This model also can provide a framework for understanding the basis and limitations of approximations like Mike Kang's electrostatic field modeling (with FLUX2D) at LANL and various lumped circuit models.

These results are in good qualitative agreement with numerical results previously found both by T. Hughes using the electromagnetic code LSP and M. Kang using FLUX-2D. Our analysis also shows that such deviations exponentially decay with radius on scale lengths of a couple inches or so for typical DARHT-2 Metglas core accelerator cell parameters. There appears to be an $\sim 20-35 \%$ difference between the predicted values of the decay length as compared with the (smaller) value inferred from fits to measurements on the LBNL test stand and numerical simulation of the actual DARHT-2 cell configuration. A more accurate analytical solution would involve a more complicated eigenfunction/eigenvalue solution for the actual cell geometry that would take into account, for example, gap separations which vary from core-to-core and similar variations amongst the different cores in the radial separation between each core's outer radius and the outer accelerator cell enclosure.

We thank Lou Reginato and Will Waldron their efforts in making extensive measurements on the LBNL Core Voltage Test Stand and being always willing to continually adapt their setup to our constantly changing wishes. We also are pleased to acknowledge many useful discussions with Mike Kang of LANL and Tom Hughes of MRC. 